Sharif University of Technology
Scientia Iranica
Transactions E: Industrial Engineering
hCIENTIA

\title{
A Malmquist productivity index with the directional distance function and uncertain data
}

\author{
N. Aghayi ${ }^{\mathrm{a}, *}$, M. Tavana ${ }^{\mathrm{b}, \mathrm{c}}$, and B. Maleki ${ }^{\mathrm{a}}$ \\ a. Department of Mathematics, Ardabil Branch, Islamic Azad University, Ardabil, Iran. \\ b. Department of Business Systems and Analytics, Lindback Distinguished Chair of Information Systems and Decision Sciences, La \\ Salle University, Philadelphia, PA 19141, USA. \\ c. Department of Business Information Systems, Faculty of Business Administration and Economics, University of Paderborn, \\ D-33098 Paderborn, Germany.
}

Received 25 September 2017; received in revised form 13 February 2018; accepted 16 July 2018

\author{
KEYWORDS \\ Data envelopment \\ analysis; \\ Malmquist \\ productivity index; \\ Interval approach; \\ Directional distance \\ function; \\ Undesirable outputs.
}

\begin{abstract}
In the present study, by using the directional distance function with undesirable interval outputs, the Malmquist Productivity Index (MPI) and integrated Data Envelopment Analysis (DEA) are employed for evaluating the function of Decision-Making Units (DMUs). The MPI calculation is performed to compare the efficiency of the DMUs in distinct time periods. The uncertainty inherent in real-world problems is considered by using the best- and worst-case scenarios, defining an interval for the MPI, and reflecting the DMUs' advancement or regress. The optimal solution of the robust model lies in the efficiency interval, i.e., it is always equal to or less than the optimal solution in the optimistic case and equal to or greater than the optimal solution in the pessimistic case. This study also presented a case study in the banking industry to demonstrate the applicability and efficacy of the proposed integrated approach.
\end{abstract}

(C) 2019 Sharif University of Technology. All rights reserved.

\section{Introduction}

Building on the ideas of Farrell and twenty years after his pioneering work [1], Charnes et al. [2] developed the DEA technique, which is a non-parametric method for measuring the efficiency of a set of Decision-Making Units (DMUs) that use multiple inputs to produce multiple outputs. The model presented by Charnes et al. [2] with a constant return to scale is called the CCR model. In order to devise a new BCC model, some changes were applied to the CCR model by Banker et al. [3]. In DEA models, an inefficient

\footnotetext{
*. Corresponding author.

E-mail addresses: nazila.aghayi@gmail.com (N. Aghayi); tavana@lasalle.edu(M.Tavana); hodamaleki62@yahoo.com (B. Maleki)
}

doi: $10.24200 /$ sci. 2018.5259 .1173
DMU could improve its efficiency by increasing output levels (produced results) or decreasing input levels (consumed resources). In the real world, however, a DMU may have both desirable and undesirable outputs at the same time. Pittman et al. [4] investigated the use of undesirable outputs to perform an efficient evaluation based on the extended model of Caves et al. [5] so that the efficiency of DMUs could be measured in the presence of desirable and undesirable outputs. Further, Ardabili et al. [6] applied undesirable indexes to evaluate DMUs. In addition to comparing the relative performance of a set of DMUs in a specific period, conventional DEA can also be used to calculate the productivity changes of a DMU over time. The Malmquist Productivity Index (MPI) is a model capable of computing the relative performance of a DMU in different time periods. Initially termed as a quality productivity index, the MPI was first introduced in 1953 by Malmquist [7] as ratios of distance functions 
for analyzing the consumption of resources in the production. This productivity index was later applied to productivity measurement and analysis. There are several decompositions of the MPI in the literature, with the most popular one proposed by Fare et al. [8]. Now, many researchers consider some degree of data uncertainty in different time periods.

In recent years, numerous studies have considered combining data uncertainty in optimized models. However, most of them have relied on complicated nonlinear models. Due to the general assumption that the input data are absolutely known, the effects of this issue on flexibility and optimality of models have not been considered. Hence, several constraints appear to be violated once the data take values other than their nominal values; the results that have previously obtained optimal solution-when nominal data have been investigated-may not remain optimal or even feasible anymore. In the early 1970s, a linear optimization model, which provided a flexible solution for input data and could take on any value from an interval, was proposed by Soyster [9]. This approach, however, deviated considerably from the nominal problem optimality to ensure the robustness of the solution.

In the current paper, to evaluate the MPI of the DMUs in the best- and the worst-case scenarios, the optimistic and pessimistic models are presented by considering desirable and undesirable interval output data; therefore, the interval has been accomplished for the MPI of the DMUs. In addition, all DMUs are divided into six categories. At the end of intervals, since the maximum coincidence may not be obtained, the considered interval method may not be able to carry out an accurate analysis. Therefore, the purpose of this study is to present an optimized method for computing the MPI of the DMUs. In so doing, the flexibility of the obtained solution will no longer be problematic because the accuracy of the solution is guaranteed by the level of conservatism.

\subsection{Background}

Most DEA papers in recent years have been proposed by researchers to measure the efficiency of DMUs. Each of these methods has been developed based on earlier hypotheses. There are generally two approaches in the DEA literature to confronting undesirable outputs. One approach relies on an indirect method presented by Seiford and Zhu [10] following the changes applied to the model suggested by Charnes et al. [2]. Another method is based on the direct approach of Chambers et al. [11], which was developed by Chung et al. [12].

Constrictions of other methods have been somehow eliminated by applying a novel model derived from the model of Shepherd [13]; in addition, efficient calculations could be carried out in the case of undesirable outputs based on the directional distance function [14]. Iftikhar et al. [15] used undesirable data in DEA and estimated energy and $\mathrm{CO}_{2}$ emissions. In order to determine the marginal rates of substitution in data envelopment analysis with undesirable outputs, Khoshandam et al. [16] presented a new approach. The MPI facilitates the decomposition of productivity into its two major components, i.e., technological change and technical efficiency change. In other words, Malmquist analysis allows separating shifts in the efficiency frontier (technological change) from improvements in the efficiency associated with the frontier (technical efficiency change). These two components are different from each other in terms of both basis and analysis and, therefore, require different policy measures. The product of technological change and technical efficiency change is the total factor productivity change, which is measured by the MPI. A wealth of information can be derived from the MPI. The MPI not only reveals patterns of productivity change and presents a new interpretation along with the managerial implication of each Malmquist component, but also identifies strategic orientations of an organization in past time periods for proper selection in future periods.

Barnabé [17] proposed the application of the Malquist productivity index to power factories. He showed how this index could be used to evaluate the costs of productivity and, also, productivity changes. Sueyoshi et al. [18] measured the efficiency of sustainability enhancement in China. Sueyoshi and Goto [19] used the MPI for the environmental assessment of petroleum companies. The MPI was also employed by Fuentes and Lillo-Banuls [20] to help perform efficiency evaluation of tax offices in Spain from 2004 to 2006. By applying the MPI, Yu et al. [21] carried out an assessment of the eco-efficiency performance of the pulp and paper industry in China. Kao [22] measured the MPI for parallel production systems. Maroto and Zofio [23] utilized the Malmquist approach to provide a model for accessibility gains and road transport infrastructure in Spain during the 1995-2005 period. In addition, the overall profit of MPI with interval data and fuzzy was investigated by Emrouznejad et al. [24].

Although there are various methods for optimization with uncertainty, most of them have problems. In order to confront data uncertainty, a fuzzy approach was adopted by Wanke et al. [25]. He also calculated the efficiency of banks. Sorting the genetic algorithm with uncertain data, Mashayekhi and Omrani [26] used a fuzzy approach, too. Aghayi [27] measured cost efficiency with fuzzy data in DEA. A framework was presented by Toloo et al. [28], where DEA was used to measure the overall profit efficiency with interval data. The efficiency of banks with interval data was calculated by Hatami-Marbini et al. [29]. Salehpour 
and Aghayi [30] strived to calculate the highest revenue efficiency with price uncertainty. In order to find a solution for minimizing the worst-case performance with uncertain data, Kouvelis and $\mathrm{Yu}$ [31] considered a robust optimization model.

For achieving a robust optimal solution, various approaches were presented by Ben-Tal and Nemirovski [32] and El-Ghaoui and Lebret [33]. However, their methods made the robust problem more complex. A robust optimization method, presented by Bertsimas and Sym [34] for linear problems, made the problem more tractable by adjusting the conservatism degree. Zahedi-Seresht et al. [35] ranked the DMUs based on sensitivity analysis by robust optimization. Yousefi et al. [36] ranked the sustainable supply chains using network goal programming DEA model and robust fuzzy optimization. For calculating the efficiency of vehicle routing operators, ChungCheng [37] presented a robust method. In order to measure the technical efficiency of potato production in Iran, Mardani and Salarpour [38] employed a robust method. Aghayi et al. [39] investigated the efficiency of robust measurement with typical weights, data uncertainty, and various conservatism degrees. To evaluate the efficiency measurement of DMUs with undesirable outputs, Aghayi and Maleki [40] applied a robust method.

\subsection{Organization}

The rest of the paper is organized as follows. Section 2 calculates the MPI of the directional distance function model with undesirable outputs and data uncertainty. In Section 3, by considering interval and robust methods, a model is presented that calculates the MPI based on the directional distance function with undesirable outputs and data uncertainty. Finally, to demonstrate the applicability of the proposed methods, a numerical example is explained in Section 4, which is followed by conclusions in Section 5.

\section{Computing the MPI of DMUs with undesirable outputs under data certainty based on the directional distance function model}

Here, it is assumed that there are $n$ DMUs with constant inputs, $s$ desirable outputs, and $l$ undesirable outputs; the desirable and undesirable output vectors for $D M U_{j}$ are $y_{j}=\left(y_{1 j}, \cdots, y_{s j}\right)$ and $b_{j}=$ $\left(b_{1 j}, \cdots, b_{l j}\right)$, respectively. To evaluate the efficiency of $D M U_{o}$, the following model was presented by Zanella et al. [14]:

$\beta_{o}^{*}=\max \beta$,

s.t.:

$$
\begin{array}{ll}
\sum_{j=1}^{n} b_{k j} \lambda_{j} \leq b_{k o}-\beta g_{b}, & k=1, \cdots, l, \\
\sum_{j=1}^{n} y_{r j} \lambda_{j} \geq y_{r o}+\beta g_{y}, & r=1, \cdots, s, \\
\sum_{j=1}^{n} \lambda_{j}=1, & \\
\lambda_{j} \geq 0, & j=1, \cdots, n,
\end{array}
$$

where $g=\left(-g_{b}, g_{y}\right)=\left(-b_{k o}, y_{r o}\right) . \beta_{o}^{*} \geq 0$ in all cases; if $\beta_{o}^{*}=0$, then $D M U_{o}$ is efficient; otherwise, $D M U_{o}$ is inefficient. Therefore, the efficiency of the evaluated unit can be computed as $\rho_{o}=\frac{1}{1+\beta_{o}^{*}}$, where $0<\rho_{o} \leq 1$. For more information on Model (1), refer to Aghayi and Maleki [40]. Model (2) is the dual of Model (1):

$$
\begin{aligned}
& \theta_{o}^{*}=\min -\sum_{r=1}^{s} y_{r o} u_{r}+\sum_{k=1}^{l} b_{k o} d_{k}+v, \\
& \text { s.t.: } \\
& \sum_{r=1}^{s} g_{y} u_{r}+\sum_{k=1}^{l} g_{b} d_{k}=1, \\
& -\sum_{r=1}^{s} y_{r j} u_{r}+\sum_{k=1}^{l} b_{k j} d_{k}+v \geq 0, \quad j=1, \cdots, n, \\
& u_{r} \geq 0, \quad r=1, \cdots, s, \\
& d_{k} \geq 0, \quad k=1, \cdots, l .
\end{aligned}
$$

Definition 1. In Model (2), if $\theta_{o}^{*}=0$, then $D M U_{o}$ is efficient.

Definition 2. In Model (2), the efficiency of $D M U_{o}$ can be calculated by $\phi_{o}^{*}=\frac{1}{1+\theta^{*}}$. Thus, $D M U_{o}$ is efficient if $\phi_{o}^{*}=1$. If $\phi_{o}^{*}<1$, then $D M U_{o}$ is inefficient.

In this section, a model for calculating the MPI is presented. Malmquist analysis relies on the implementation of distance functions. Distance functions based on two distinct time periods as $\theta_{o}^{t}\left(x^{t+1}, y^{t+1}\right)$ and $\theta_{o}^{t+1}\left(x^{t}, y^{t}\right)$ are defined, where $\theta_{o}^{t+1}$ represents the distance function associated with the frontier at time $t+1$, and $\left(x^{t+1}, y^{t+1}\right)$ denotes input and output vectors at time $t+1$. The function $\theta_{o}^{t+1}\left(x^{t}, y^{t}\right)$ evaluates the input-output combination in period $t$ in relation to technology in period $t+1$, whereas the function $\theta_{o}^{t}\left(x^{t+1}, y^{t+1}\right)$ evaluates the observed inputoutput combination in period $t+1$ in relation to technology in period $t$. Distance functions for an input-output vector in a specific year in relation to the 
frontier in the same year are represented by $\theta_{o}^{t}\left(x^{t}, y^{t}\right)$ and $\theta_{o}^{t+1}\left(x^{t+1}, y^{t+1}\right)$ for years $t$ and $t+1$, respectively. Hence, the directional distance function model with undesirable outputs for the Malmquist index is given as follows:

DMU at time $t+1$ and the frontier at time $t$ :

$$
\theta_{o}^{p}\left(y_{o}^{p}, b_{o}^{p} \mid p=t, t+1\right)=\min -\sum_{r=1}^{s} y_{r o}^{p} u_{r}+\sum_{k=1}^{l} b_{k o}^{p} d_{k}+v,
$$

s.t.:

$$
\begin{aligned}
& \sum_{r=1}^{s} g_{y} u_{r}+\sum_{k=1}^{l} g_{b} d_{k}=1, \\
& -\sum_{r=1}^{s} y_{r j}^{p} u_{r}+\sum_{k=1}^{l} b_{k j}^{p} d_{k}+v \geq 0, \quad j=1, \cdots, n, \\
& u_{r} \geq 0, \quad r=1, \cdots, s, \quad d_{k} \geq 0, \quad k=1, \cdots, l .
\end{aligned}
$$

DMU at time $t$ and the frontier at time $t+1$ :

$$
\begin{gathered}
\theta_{o}^{p}\left(y_{o}^{q}, b_{o}^{q} \mid q, p=t, t+1, p \neq q\right)=\min \\
-\sum_{r=1}^{s} y_{r o}^{q} u_{r}+\sum_{k=1}^{l} b_{k o}^{q} d_{k}+v
\end{gathered}
$$

s.t.:

$$
\begin{aligned}
& \sum_{r=1}^{s} g_{y} u_{r}+\sum_{k=1}^{l} g_{b} d_{k}=1, \\
& -\sum_{r=1}^{s} y_{r j}^{p} u_{r}+\sum_{k=1}^{l} b_{k j}^{p} d_{k}+v \geq 0, \quad j=1, \cdots, n, \\
& u_{r} \geq 0, \quad r=1, \cdots, s, \quad d_{k} \geq 0, \quad k=1, \cdots, l .
\end{aligned}
$$

In Model (3), where the DMU is at time $t+1$ and the frontier is at time $t, b^{p}$ and $y^{p}$ represent matrices corresponding to the desirable and undesirable outputs of the data observed in period $p$, respectively. Thus, Model (3) is solved for $p=t$ and $p=t+1$. Model (4), where the DMU is at time $t$ and the frontier is at time $t+1$, is also solved for $q, p=t, t+1, p \neq q$.

Definition 3. If the value of the objective function in Models (3) and (4) is zero, then $D M U_{o}$ is efficient.

Definition 4. The efficiency score of $D M U_{o}$ in Models (3) and (4) is calculated by $\phi_{o}^{*}=\frac{1}{1+\theta_{o}^{*}}$. Hence, $D M U_{o}$ is efficient if $\phi_{o}^{*}=1$; if $\phi_{o}^{*}<1$, then $D M U_{o}$ is inefficient.

When the MPI is to be calculated, there are only two sources of productivity growth, i.e., efficiency change $(\mathrm{EFCH})$ and technical change $(\mathrm{TCH})$, if the production process has constant returns to scale. The geometric mean of these two sources is usually used to calculate the MPI. On the other hand, if the production process exhibits variable returns to scale, the effects of two additional sources of productivity growth, namely pure technical efficiency (PTECH) and scale efficiency (SECH), are also taken into consideration. As proposed by Ray and Desli [41], calculation of the MPI under variable returns to scale with undesirable outputs in relation to any technology in period $t$ or $t+1$ can be performed as follows:

$$
\begin{aligned}
M_{o}= & \sqrt{\frac{\phi_{v}^{t}\left(y_{o}^{t+1}, b_{o}^{t+1}\right)}{\phi_{v}^{t}\left(y_{o}^{t}, b_{o}^{t}\right)} \times \frac{\phi_{v}^{t+1}\left(y_{o}^{t+1}, b_{o}^{t+1}\right)}{\phi_{v}^{t+1}\left(y_{o}^{t}, b_{o}^{t}\right)}} \\
& \times \sqrt{\frac{S E^{t}\left(y_{o}^{t+1}, b_{o}^{t+1}\right)}{S E^{t}\left(y_{o}^{t}, b_{o}^{t}\right)} \times \frac{S E^{t+1}\left(y_{o}^{t+1}, b_{o}^{t+1}\right)}{S E^{t+1}\left(y_{o}^{t}, b_{o}^{t}\right)}},
\end{aligned}
$$

where $\phi_{v}^{t}$ measures the productivity growth between periods $t$ and $t+1$ by using the technology of period $t$ as technology, and $\phi_{v}^{t+1}$ measures the same value by employing the technology of period $t+1$ as the reference technology under Variable Returns to Scale (VRS). Besides, $S E^{t}$ and $S E^{t+1}$ represent the scale efficiencies when the frontier is in periods $t$ and $t+1$, respectively, and are given by:

$$
\begin{aligned}
& S E^{t}\left(y_{o}^{t+1}, b_{o}^{t+1}\right)=\frac{\phi_{c}^{t}\left(y_{o}^{t+1}, b_{o}^{t+1}\right)}{\phi_{v}^{t}\left(y_{o}^{t+1}, b_{o}^{t+1}\right)}, \\
& S E^{t+1}\left(y_{o}^{t+1}, b_{o}^{t+1}\right)=\frac{\phi_{c}^{t+1}\left(y_{o}^{t+1}, b_{o}^{t+1}\right)}{\phi_{v}^{t+1}\left(y_{o}^{t+1}, b_{o}^{t+1}\right)}, \\
& S E^{t}\left(y_{o}^{t}, b_{o}^{t}\right)=\frac{\phi_{c}^{t}\left(y_{o}^{t}, b_{o}^{t}\right)}{\phi_{v}^{t}\left(y_{o}^{t}, b_{o}^{t}\right)}, \\
& S E^{t+1}\left(y_{o}^{t}, b_{o}^{t}\right)=\frac{\phi_{c}^{t+1}\left(y_{o}^{t}, b_{o}^{t}\right)}{\phi_{v}^{t+1}\left(y_{o}^{t}, b_{o}^{t}\right)},
\end{aligned}
$$

where $\phi_{c}^{t}$ and $\phi_{c}^{t+1}$ assess the productivity growth under constant returns to scale in periods $t$ and $t+1$, respectively. The results are interpreted as follows:

1. $M_{o}>1$ shows productivity increase or progress;

2. $M_{o}<1$ reveals productivity decrease;

3. $M_{o}=1$ reflects unchanged productivity during the two periods.

\section{Calculating the MPI of DMUs with undesirable outputs with data uncertainty based on the directional distance function model}

Here, it is considered that there are $n$ DMUs with constant inputs, $s$ desirable interval outputs, and $l$ 
undesirable interval outputs; the desirable and undesirable output vectors for $D M U_{j}$ are $\bar{y}_{j}=\left(\bar{y}_{1 j}, \cdots, \bar{y}_{s j}\right)$ and $\bar{b}_{j}=\left(\bar{b}_{1 j}, \cdots, \bar{b}_{l j}\right)$, respectively, where $\bar{y}_{j} \in$ $\left[y_{j}^{L}, y_{j}^{U}\right]$ and $\bar{b}_{j} \in\left[b_{j}^{L}, b_{j}^{U}\right] . \quad y_{j}^{L}$ and $b_{j}^{L}$, respectively, indicate the lower bound of desirable and undesirable outputs for $D M U_{j}$. For calculating MPI for $D M U_{o}$ with data uncertainty, the following models can be used:

DMU at time $t+1$ and the frontier at time $t$ :

$$
\bar{\theta}_{o}^{p}\left(\bar{y}_{o}^{p}, \bar{b}_{o}^{p} \mid p=t, t+1\right)=\min -\sum_{r=1}^{s} \bar{y}_{r o}^{p} u_{r}+\sum_{k=1}^{l} \bar{b}_{k o}^{p} d_{k}+v
$$

s.t.:

$\sum_{r=1}^{s} g_{y} u_{r}+\sum_{k=1}^{l} g_{b} d_{k}=1$

$-\sum_{r=1}^{s} \bar{y}_{r j}^{p} u_{r}+\sum_{k=1}^{l} \bar{b}_{k j}^{p} d_{k}+v \geq 0, \quad j=1, \cdots, n$,

$u_{r} \geq 0, \quad r=1, \cdots, s, \quad d_{k} \geq 0, \quad k=1, \cdots, l$.

DMU at time $t$ and the frontier at time $t+1$ :

$\bar{\theta}_{o}^{p}\left(y_{o}^{q}, b_{o}^{q} \mid q, p=t, t+1, p \neq q\right)=\min$

$$
-\sum_{r=1}^{s} \bar{y}_{r o}^{q} u_{r}+\sum_{k=1}^{l} \bar{b}_{k o}^{q} d_{k}+v
$$

s.t.:

$\sum_{r=1}^{s} g_{y} u_{r}+\sum_{k=1}^{l} g_{b} d_{k}=1$

$-\sum_{r=1}^{s} \bar{y}_{r j}^{p} u_{r}+\sum_{k=1}^{l} \bar{b}_{k j}^{p} d_{k}+v \geq 0, \quad j=1, \cdots, n$,

$u_{r} \geq 0, \quad r=1, \cdots, s, \quad d_{k} \geq 0, \quad k=1, \cdots, l$.

In the following, two approaches to solving Models (8) and (9) are presented: one is based on interval concepts and the other relies on robust optimization.

\subsection{Calculating MPI based on the interval approach}

In Models (8) and (9), due to the changes in input and output parameters over interval, the values of DMUs efficiency are included in the interval. The efficiency value of the DMUs for the upper and lower bounds can be calculated in the following. To calculate the efficiency value of lower bound, the DMU is evaluated in the worst-case scenario (lower bound with its desirable output and the upper bound with its undesirable output) and the other DMUs in the best-case scenario (by exploring the upper bound of desirable outputs and the lower bound of undesirable outputs). Thus, in pessimistic conditions, to measure the efficiency of $D M U_{0}$ based on the directional distance function, the following models are presented:

DMU at time $t+1$ and the frontier at time $t$ :

$\theta_{o}^{L p}\left(y_{o}^{L p}, b_{o}^{U p} \mid p=t, t+1\right)=\min -\sum_{r=1}^{s} y_{r o}^{L p} u_{r}+\sum_{k=1}^{l} b_{k o}^{U p} d_{k}+v$

s.t.:

$$
\begin{aligned}
& \sum_{r=1}^{s} g_{y} u_{r}+\sum_{k=1}^{l} g_{b} d_{k}=1, \\
& -\sum_{r=1}^{s} y_{r j}^{U p} u_{r}+\sum_{k=1}^{l} b_{k j}^{L p} d_{k}+v \geq 0, \\
& j=1, \cdots, n, \quad j \neq o, \\
& -\sum_{r=1}^{s} y_{r o}^{L p} u_{r}+\sum_{k=1}^{l} b_{k o}^{U p} d_{k}+v \geq 0, \\
& u_{r} \geq 0, \quad r=1, \cdots, s, \quad d_{k} \geq 0, \quad k=1, \cdots, l .
\end{aligned}
$$

DMU at time $t$ and the frontier at time $t+1$ :

$$
\begin{aligned}
& \theta_{o}^{L p}\left(y_{o}^{L q}, b_{o}^{U q} \mid q, p=t, t+1, p \neq q\right)=\min \\
& \quad-\sum_{r=1}^{s} y_{r o}^{L q} u_{r}+\sum_{k=1}^{l} b_{k o}^{U q} d_{k}+v \\
& \text { s.t.: } \\
& \sum_{r=1}^{s} g_{y} u_{r}+\sum_{k=1}^{l} g_{b} d_{k}=1, \\
& -\sum_{r=1}^{s} y_{r j}^{U p} u_{r}+\sum_{k=1}^{l} b_{k j}^{L p} d_{k}+v \geq 0, \\
& \quad j=1, \cdots, n, \quad j \neq o, \\
& -\sum_{r=1}^{s} y_{r o}^{L q} u_{r}+\sum_{k=1}^{l} b_{k o}^{U q} d_{k}+v \geq 0, \\
& u_{r} \geq 0, \quad r=1, \cdots, s, \quad d_{k} \geq 0, \quad k=1, \cdots, l .
\end{aligned}
$$

Definition 5. In Models (10) and (11), $D M U_{o}$ is said to be efficient under pessimistic conditions if $\theta_{o}^{L P}=0$. 
Definition 6. Efficiency measure in the lower bound is given by $\phi_{o}^{L P}=\frac{1}{1+\theta_{o}^{L P}}$. If $\phi_{o}^{L P}=1$, then $D M U_{o}$ is efficient.

Conclusion 1. The lower bound of the MPI is calculated as follows:

$$
\begin{aligned}
M^{L}= & \sqrt{\frac{\phi_{v}^{L t}\left(y_{o}^{t+1}, b_{o}^{t+1}\right)}{\phi_{v}^{U t}\left(y_{o}^{t}, b_{o}^{t}\right)} \times \frac{\phi_{v}^{L t+1}\left(y_{o}^{t+1}, b_{o}^{t+1}\right)}{\phi_{v}^{U t+1}\left(y_{o}^{t}, b_{o}^{t}\right)}} \\
& \times \sqrt{\frac{S E^{L t}\left(y_{o}^{t+1}, b_{o}^{t+1}\right)}{S E^{U t}\left(y_{o}^{t}, b_{o}^{t}\right)} \times \frac{S E^{L t+1}\left(y_{o}^{t+1}, b_{o}^{t+1}\right)}{S E^{U t+1}\left(y_{o}^{t}, b_{o}^{t}\right)}} .
\end{aligned}
$$

From an optimistic perspective, when the evaluated $\mathrm{DMU}$ is investigated in the best-case scenario, it means that the upper bound is considered in its desirable output and the lower bound is considered in its undesirable output. In addition, when the other DMUs are investigated in the worst-case scenario, it means that the lower bound is considered in desirable outputs and the upper bound is considered in undesirable outputs. For calculating the optimistic efficiency of $D M U_{o}$ according to the directional distance function, the following models can be used:

DMU at time $t+1$ and the frontier at time $t$ :

$$
\begin{array}{r}
\theta_{o}^{U p}\left(y_{o}^{U p}, b_{o}^{L p} \mid p=t, t+1\right)=\min \\
-\sum_{r=1}^{s} y_{r o}^{U p} u_{r}+\sum_{k=1}^{l} b_{k o}^{L p} d_{k}+v
\end{array}
$$

s.t.:

$$
\begin{aligned}
& \sum_{r=1}^{s} g_{y} u_{r}+\sum_{k=1}^{l} g_{b} d_{k}=1, \\
& -\sum_{r=1}^{s} y_{r j}^{L p} u_{r}+\sum_{k=1}^{l} b_{k j}^{U p} d_{k}+v \geq 0, \\
& j=1, \cdots, n, \quad j \neq o, \\
& -\sum_{r=1}^{s} y_{r o}^{U p} u_{r}+\sum_{k=1}^{l} b_{k o}^{L p} d_{k}+v \geq 0, \\
& u_{r} \geq 0, \quad r=1, \cdots, s, \quad d_{k} \geq 0, \quad k=1, \cdots, l .
\end{aligned}
$$

DMU at time $t$ and the frontier at time $t+1$ :

$$
\begin{gathered}
\theta_{o}^{U p}\left(y_{o}^{U q}, b_{o}^{L q} \mid q, p=t, t+1, p \neq q\right)=\min \\
-\sum_{r=1}^{s} y_{r o}^{U q} u_{r}+\sum_{k=1}^{l} b_{k o}^{L q} d_{k}+v
\end{gathered}
$$

$$
\begin{aligned}
& \text { s.t.: } \\
& \sum_{r=1}^{s} g_{y} u_{r}+\sum_{k=1}^{l} g_{b} d_{k}=1, \\
& -\sum_{r=1}^{s} y_{r j}^{L p} u_{r}+\sum_{k=1}^{l} b_{k j}^{U p} d_{k}+v \geq 0, \\
& \quad j=1, \cdots, n, \quad j \neq o, \\
& -\sum_{r=1}^{s} y_{r o}^{U q} u_{r}+\sum_{k=1}^{l} b_{k o}^{L q} d_{k}+v \geq 0, \\
& u_{r} \geq 0, \quad r=1, \cdots, s, \quad d_{k} \geq 0, \quad k=1, \cdots, l .
\end{aligned}
$$

Definition 7. In Models (13) and (14), $D M U_{o}$ is said to be efficient under optimistic conditions if $\theta_{o}^{U p}=0$.

Definition 8. Optimistic efficiency measures in Models (13) and (14) are given by $\phi_{o}^{U p}=\frac{1}{1+\theta_{o}^{U p}}$. If $\phi_{o}^{U p}=1$, then $D M U_{o}$ is efficient.

Conclusion 2. The upper bound of the MPI is calculated as follows:

$$
\begin{aligned}
M^{U} & =\sqrt{\frac{\phi_{v}^{U t}\left(y_{o}^{t+1}, b_{o}^{t+1}\right)}{\phi_{v}^{L t}\left(y_{o}^{t}, b_{o}^{t}\right)} \times \frac{\phi_{v}^{U t+1}\left(y_{o}^{t+1}, b_{o}^{t+1}\right)}{\phi_{v}^{L t+1}\left(y_{o}^{t}, b_{o}^{t}\right)}} \\
& \times \sqrt{\frac{S E^{U t}\left(y_{o}^{t+1}, b_{o}^{t+1}\right)}{S E^{L t}\left(y_{o}^{t}, b_{o}^{t}\right)} \times \frac{S E^{U t+1}\left(y_{o}^{t+1}, b_{o}^{t+1}\right)}{S E^{L t+1}\left(y_{o}^{t}, b_{o}^{t}\right)}} .
\end{aligned}
$$

Theorem 1. Prove $M^{L} \leq M^{U}$.

Proof. For indicating $\theta_{o}^{U p} \leq \theta_{o}^{L p}$, we must prove that, in Models (10) and (11), the values of objective function are smaller than those for Models (13) and (14), that is to say, $\theta_{o}^{L p}-\theta_{o}^{U p} \geq 0$. For optimistic and pessimistic models, the values of objective function are considered as follows:

$$
\begin{aligned}
& \theta_{o}^{L p}=-\sum_{r=1}^{s} u_{r} y_{r o}^{L p}+\sum_{k=1}^{l} d_{k} b_{k o}^{U p}+v, \\
& \theta_{o}^{U p}=-\sum_{r=1}^{s} u_{r} y_{r o}^{U p}+\sum_{k=1}^{l} d_{k} b_{k o}^{L p}+v .
\end{aligned}
$$

Now, obtaining the result of $\theta_{o}^{L P}-\theta_{o}^{U P}$, we can write:

$$
\begin{aligned}
\theta_{o}^{L p}-\theta_{o}^{U p}= & \sum_{r=1}^{s} u_{r}\left(y_{r o}^{U p}-y_{\text {ro }}^{L p}\right) \\
& +\sum_{k=1}^{l} d_{k}\left(b_{k o}^{U p}-b_{k o}^{L p}\right)+v-v .
\end{aligned}
$$


Considering the fact that $u_{r}, d_{k} \geq 0, y_{\text {ro }}^{U p} \geq y_{\text {ro }}^{L p}$, and $b_{k o}^{U p} \geq b_{k o}^{L p}$, Eq. (17) will be a positive value. Therefore, $\theta_{o}^{U p} \leq \theta_{o}^{L p} ;$ it is also obvious that $\phi_{o}^{L p} \leq \phi_{o}^{U p}$. Now, let the lower and upper bounds be given by:

$$
\begin{aligned}
M^{L}= & \sqrt{\frac{\phi_{v}^{L t}\left(y_{o}^{t+1}, b_{o}^{t+1}\right)}{\phi_{v}^{U t}\left(y_{o}^{t}, b_{o}^{t}\right)} \times \frac{\phi_{v}^{L t+1}\left(y_{o}^{t+1}, b_{o}^{t+1}\right)}{\phi_{v}^{U t+1}\left(y_{o}^{t}, b_{o}^{t}\right)}} \\
& \times \sqrt{\frac{S E^{L t}\left(y_{o}^{t+1}, b_{o}^{t+1}\right)}{S E^{U t}\left(y_{o}^{t}, b_{o}^{t}\right)} \times \frac{S E^{L t+1}\left(y_{o}^{t+1}, b_{o}^{t+1}\right)}{S E^{U t+1}\left(y_{o}^{t}, b_{o}^{t}\right)}}, \\
M^{U}= & \sqrt{\frac{\phi_{v}^{U t}\left(y_{o}^{t+1}, b_{o}^{t+1}\right)}{\phi_{v}^{L t}\left(y_{o}^{t}, b_{o}^{t}\right)} \times \frac{\phi_{v}^{U t+1}\left(y_{o}^{t+1}, b_{o}^{t+1}\right)}{\phi_{v}^{L t+1}\left(y_{o}^{t}, b_{o}^{t}\right)}} \\
& \times \sqrt{\frac{S E^{U t}\left(y_{o}^{t+1}, b_{o}^{t+1}\right)}{S E^{L t}\left(y_{o}^{t}, b_{o}^{t}\right)} \times \frac{S E^{U t+1}\left(y_{o}^{t+1}, b_{o}^{t+1}\right)}{S E^{L t+1}\left(y_{o}^{t}, b_{o}^{t}\right)}} .
\end{aligned}
$$

Since $\phi_{o}^{L p} \leq \phi_{o}^{U p}$, it is obvious that $M^{L} \leq M^{U}$.

Conclusion 3. If $M_{o}^{U}$ and $M_{o}^{L}$ are the upper and lower bounds of the MPI, respectively, as obtained by the directional distance function, then $\bar{M}_{o} \in$ $\left[M_{o}^{L}, M_{o}^{U}\right]$.

By considering that the MPI of each DMU lies at an interval, the DMUs can be divided into six categories:

a. DMUs with constant productivity, i.e., $E^{o}=$ $\left\{D M U_{j}: M_{j}^{U}=M_{j}^{L}=1\right\}$;

b. All DMUs with increasing productivity and progress in the pessimistic case, i.e., $E^{++}=\left\{D M U_{j}: 1<\right.$ $\left.M_{j}^{L} \leq M_{j}^{U}\right\}$

c. All DMUs with decreasing productivity and regress in the optimistic case, i.e., $E^{--}=\left\{D M U_{j}: M_{j}^{L} \leq\right.$ $\left.M_{j}^{U}<1\right\}$;

d. All DMUs with increasing productivity in the optimistic case and unchanged productivity in the pessimistic case, i.e., $E^{+}=\left\{D M U_{j}: M_{j}^{L}=\right.$ $\left.1, M_{j}^{U}>1\right\}$

e. All DMUs with decreasing productivity in the pessimistic case and unchanged productivity in the optimistic case, i.e., $E^{-}=\left\{D M U_{j}: M_{j}^{L}<\right.$ $\left.1, M_{j}^{U}=1\right\}$

f. All DMUs with increasing productivity in the optimistic case and decreasing productivity in the pessimistic case, i.e., $E=\left\{D M U_{j}: M_{j}^{L}<1<\right.$ $\left.M_{j}^{U}\right\}$.

\subsection{Calculating the MPI based on the robust approach}

There are $n$ DMUs, $D M U_{j}(j=1, \cdots, n)$, with desirable and undesirable interval outputs. $\left|J_{j}^{y}\right|$ and $\left|J_{j}^{b}\right|$ are the number of desirable and undesirable outputs in the $j$ th constraint, respectively. The role of $\left|\gamma_{j}^{y}\right|$ and $\left|\gamma_{j}^{b}\right|$ parameters $\left(\gamma_{j}^{y} \in\left[0,\left|J_{j}^{y}\right|\right]\right.$ and $\left.\gamma_{j}^{b} \in\left[0,\left|J_{j}^{b}\right|\right]\right)$ is adapting the robustness of the considered method in the conservation level of the solution. Here, the purpose is to ensure that the obtained solution will remain flexible when $\gamma_{j}^{y}$ and $\gamma_{j}^{b}$ are changed by $\left(\gamma_{j}^{y}-\right.$ $\left.\left[\gamma_{j}^{y}\right]\right)\left(y_{t j}^{U p}-y_{t j}^{L p}\right)$ and $\left(\gamma_{j}^{b}-\left[\gamma_{j}^{b}\right]\right)\left(b_{t j}^{U p}-b_{t j}^{L p}\right)$. In other words, the behavior of data related to the desirable and undesirable outputs highly depends on this assumption that changes in a subset of coefficients adversely affect the solution. Therefore, we can be ensured that the optimal robust solution remains feasible. For efficiency assessment, the following robust models are presented:

DMU at time $t+1$ and the frontier at time $t$ :

$$
\begin{gathered}
\theta_{o}^{R p}\left(y_{o}^{U p}, b_{o}^{L p} \mid p=t, t+1\right)=\min \\
-\sum_{r=1}^{s} y_{r o}^{U p} u_{r}+\sum_{k=1}^{l} d_{k} b_{k o}^{L p}+v \\
+\max _{C_{o}^{y}}\left\{\sum_{r \in S_{o}^{Y}} u_{r}\left(y_{r o}^{U p}-y_{r o}^{L p}\right)\right. \\
\left.+\left(\gamma_{o}^{y}-\left[\gamma_{o}^{y}\right]\right) u_{t o}^{y}\left(y_{r o}^{U p}-y_{r o}^{L p}\right)\right\} \\
+\max _{C_{o}^{b}}\left\{\sum_{k \in S_{o}^{b}} d_{k}\left(b_{k o}^{U p}-b_{k o}^{L p}\right)\right. \\
\left.+\left(\gamma_{o}^{b}-\left[\gamma_{o}^{b}\right]\right) d_{t o}^{b}\left(b_{t o}^{U p}-b_{t o}^{L p}\right)\right\},
\end{gathered}
$$

s.t.:

$$
\begin{aligned}
& \sum_{k=1}^{l} g_{b} d_{k}+\sum_{r=1}^{s} g_{y} u_{r}=1, \\
& \sum_{r=1}^{s} y_{r j}^{L p} u_{r}-\sum_{k=1}^{l} b_{k j}^{U p} d_{k}+\max _{c_{j}^{y}}\left\{\sum_{r \in S_{j}^{Y}} u_{r}\left(y_{r j}^{U p}-y_{r j}^{L p}\right)\right. \\
& \left.\quad+\left(\gamma_{o}^{y}-\left[\gamma_{o}^{y}\right]\right) u_{t j}^{y}\left(y_{t j}^{U p}-y_{t j}^{L p}\right)\right\} \\
& \quad+\max _{c_{j}^{b}}\left\{\sum_{k \in S_{j}^{b}}^{l} d_{k}\left(b_{k j}^{U p}-b_{k j}^{L p}\right)\right. \\
& \left.\quad+\left(\gamma_{j}^{b}-\left[\gamma_{j}^{b}\right]\right) d_{t j}^{b}\left(b_{t j}^{U p}-b_{t j}^{L p}\right)\right\}-v \leq 0
\end{aligned}
$$




$$
\begin{gathered}
j=1, \cdots, n, \quad j \neq o \\
-\sum_{r=1}^{s} y_{r o}^{U p} u_{r}+\sum_{k=1}^{l} d_{k} b_{k o}^{L p}+v \\
+\max _{C_{o}^{y}}\left\{\sum_{r \in S_{o}^{Y}} u_{r}\left(y_{r o}^{U p}-y_{r o}^{L p}\right)\right. \\
\left.+\left(\gamma_{o}^{y}-\left[\gamma_{o}^{y}\right]\right) u_{t o}^{y}\left(y_{r o}^{U p}-y_{r o}^{L p}\right)\right\} \\
+\max _{C_{o}^{b}}\left\{\sum_{k \in S_{o}^{b}} d_{k}\left(b_{k o}^{U p}-b_{k o}^{L p}\right)\right. \\
\left.+\left(\gamma_{o}^{b}-\left[\gamma_{o}^{b}\right]\right) d_{t o}^{b}\left(b_{t o}^{U p}-b_{t o}^{L p}\right)\right\} \geq 0
\end{gathered}
$$

$$
\begin{aligned}
& \left.+\left(\gamma_{o}^{y}-\left[\gamma_{o}^{y}\right]\right) u_{t j}^{y}\left(y_{t j}^{U p}-y_{t j}^{L p}\right)\right\} \\
& +\max _{c_{j}^{b}}\left\{\sum_{k \in S_{j}^{b}}^{l} d_{k}\left(b_{k j}^{U p}-b_{k j}^{L p}\right)\right. \\
& \left.+\left(\gamma_{j}^{b}-\left[\gamma_{j}^{b}\right]\right) d_{t j}^{b}\left(b_{t j}^{U p}-b_{t j}^{L p}\right)\right\}-v \leq 0, \\
& j=1, \cdots, n, \quad j \neq o, \\
& -\sum_{r=1}^{s} y_{r o}^{U q} u_{r}+\sum_{k=1}^{l} d_{k} b_{k o}^{L q}+v \\
& +\max _{C_{o}^{y}}\left\{\sum_{r \in S_{o}^{Y}} u_{r}\left(y_{r o}^{U q}-y_{r o}^{L q}\right)\right. \\
& \left.+\left(\gamma_{o}^{y}-\left[\gamma_{o}^{y}\right]\right) u_{\text {to }}^{y}\left(y_{\text {ro }}^{U q}-y_{\text {ro }}^{L q}\right)\right\} \\
& +\max _{C_{o}^{b}}\left\{\sum_{k \in S_{o}^{b}} d_{k}\left(b_{k o}^{U q}-b_{k o}^{L q}\right)\right. \\
& \left.+\left(\gamma_{o}^{b}-\left[\gamma_{o}^{b}\right]\right) d_{t o}^{b}\left(b_{t o}^{U q}-b_{t o}^{L q}\right)\right\} \geq 0 \\
& u_{r} \geq 0, \quad r=1, \cdots, s, \quad d_{k} \geq 0, \quad k=1, \cdots, l .
\end{aligned}
$$

where:

$$
\begin{aligned}
C_{j}^{y} & =\left\{S_{j}^{y} \cup\left\{t_{j}^{y}\right\}\left|S_{j}^{y} \subseteq J_{j}^{y},\right| S_{j}^{y} \mid\right. \\
& \left.=\left[\gamma_{j}^{y}\right], t_{j}^{y} \in\left(t_{j}^{y}-S_{j}^{y}\right)\right\}, \quad j=1, \cdots, n, \\
C_{j}^{b} & =\left\{S_{j}^{b} \cup\left\{t_{j}^{b}\right\}\left|S_{j}^{b} \subseteq J_{j}^{b},\right| S_{j}^{b} \mid\right. \\
& \left.=\left[\gamma_{j}^{b}\right], t_{j}^{b} \in\left(t_{j}^{b}-S_{j}^{b}\right)\right\}, \quad j=1, \cdots, n,
\end{aligned}
$$

where $t_{j}$ and $S_{j}$ are related to the data with and without perturbation, respectively. Now, if it is assumed that $\gamma_{j}^{b}=0$ and $\gamma_{j}^{y}=0$, then Models (19) and (20) are equivalent to Models (13) and (14), respectively; likewise, if $\gamma_{j}^{b}=\left|J_{j}^{b}\right|$ and $\gamma_{j}^{y}=\left|J_{j}^{y}\right|$, then Models (19) and (20) are equivalent to Models (10) and (11), respectively. After all, since Models (20) and (21) are nonlinear and difficult to solve, they can be transformed into the following linear models by applying the approach proposed by Bertsimas and Sim [34], where $z_{j}$ denotes total data perturbations. Suppose that: 


$$
\begin{aligned}
\alpha_{j}^{y}= & \max _{c_{j}^{y}}\left\{\sum_{r \in S_{j}^{Y}} u_{r}\left(y_{r j}^{U p}-y_{r j}^{L p}\right)\right. \\
& \left.+\left(\gamma_{o}^{y}-\left[\gamma_{o}^{y}\right]\right) u_{t j}^{y}\left(y_{t j}^{U p}-y_{t j}^{L p}\right)\right\}, \\
j= & 1, \cdots, n, \\
\alpha_{j}^{b}= & \max _{c_{j}^{b}}\left\{\sum_{k \in S_{j}^{b}}^{l} d_{k}\left(b_{k j}^{U p}-b_{k j}^{L p}\right)\right. \\
& \left.+\left(\gamma_{j}^{b}-\left[\gamma_{j}^{b}\right]\right) d_{t j}^{b}\left(b_{t j}^{U p}-b_{t j}^{L p}\right)\right\}, \\
j= & 1, \cdots, n .
\end{aligned}
$$

In order to obtain a linear form of Model (19), Models (23) and (24) respectively correspond to desirable and undesirable outputs in Constraint (19a):

$$
\begin{aligned}
\alpha_{j}^{y}= & \max _{c_{j}^{y}}\left\{\sum_{r \in S_{j}^{Y}} u_{r}\left(y_{r j}^{U p}-y_{r j}^{L p}\right)\right. \\
& \left.+\left(\gamma_{o}^{y}-\left[\gamma_{o}^{y}\right]\right) u_{t j}^{y}\left(y_{t j}^{U p}-y_{t j}^{L p}\right)\right\},
\end{aligned}
$$

s.t.:

$$
\sum_{j \in\left|J_{j}^{y}\right|} z_{j}^{y} \leq \gamma_{j}^{Y}
$$

$$
0 \leq z_{j} \leq 1
$$

$$
\begin{aligned}
\alpha_{j}^{b}= & \max _{c_{j}^{b}}\left\{\sum_{k \in S_{j}^{b}}^{l} d_{k}\left(b_{k j}^{U p}-b_{k j}^{L p}\right)\right. \\
& \left.+\left(\gamma_{j}^{b}-\left[\gamma_{j}^{b}\right]\right) d_{t j}^{b}\left(b_{t j}^{U p}-b_{t j}^{L p}\right)\right\}
\end{aligned}
$$

s.t.:

$$
\sum_{j \in\left|J_{j}^{b}\right|} z_{j}^{b} \leq \gamma_{j}^{b},
$$

$$
0 \leq z_{j} \leq 1,
$$

where $z_{j}$ shows the sum total of data fluctuations. Now, taking $p_{r j}$ and $q_{k j}$ as dual variables of Constraints (23a) and (24a), respectively, Constraint (19b) can be rewritten as follows:

$$
\begin{aligned}
& \sum_{r=1}^{s} u_{r} y_{r j}^{L p}+z_{j}^{y} \gamma_{j}^{y}+\sum_{r=1}^{s} p_{r j}-\sum_{k=1}^{l} d_{k} b_{k j}^{U p}+z_{j}^{b} \gamma_{j}^{b} \\
& \quad+\sum_{k=1}^{l} q_{k j}-v \leq 0, \quad j=1, \cdots, n, \quad j \neq o, \\
& z_{j}^{y}+p_{r j} \geq u_{r}\left(y_{r j}^{U p}-y_{r j}^{L p}\right), \\
& r=1, \cdots, s, \quad j=1, \cdots, n, \\
& z_{j}^{b}+q_{k j} \geq d_{k}\left(b_{k j}^{U p}-b_{k j}^{L p}\right), \\
& k=1, \cdots, l, \quad j=1, \cdots, n, \\
& u_{r} \geq 0, \quad \quad r=1, \cdots, s, \\
& d_{k} \geq 0, \quad k=1, \cdots, l, \\
& z_{j}^{y} \geq 0, \quad z_{j}^{b} \geq 0, \quad j=1, \cdots, n, \\
& p_{r j} \geq 0, \quad j=1, \cdots, n, \quad r=1, \cdots, s, \\
& q_{k j} \geq 0, \quad j=1, \cdots, n, \quad k=1, \cdots, l .(25)
\end{aligned}
$$

By applying the strong duality theorem and, also, considering the fact that Models (23) and (24) are feasible and bounded, it can be concluded that their corresponding dual problem is feasible and bounded, too.

Model (19) can be converted into a linear form so that Model (26) can be obtained as follows:

DMU at time $t+1$ and the frontier at time $t$ :

$$
\begin{aligned}
& \theta_{o}^{R p}\left(y_{o}^{U p}, b_{o}^{L p} \mid p=t, t+1\right)=\min -\sum_{r=1}^{s} u_{r} y_{r o}^{U p} \\
& +z_{o}^{y} \gamma_{o}^{y}+\sum_{r=1}^{s} p_{r o}+\sum_{k=1}^{l} d_{k} b_{k o}^{L p}+z_{o}^{b} \gamma_{o}^{b} \\
& +\sum_{k=1}^{l} q_{k o}+v
\end{aligned}
$$

s.t.:

$$
\begin{aligned}
& \sum_{r=1}^{s} u_{r} g_{y}+\sum_{k=1}^{l} d_{k} g_{b}=1 \\
& \sum_{r=1}^{s} u_{r} y_{r j}^{L p}+z_{j}^{y} \gamma_{j}^{y}+\sum_{r=1}^{s} p_{r j}-\sum_{k=1}^{l} d_{k} b_{k j}^{U p}+z_{j}^{b} \gamma_{j}^{b} \\
& \quad+\sum_{k=1}^{l} q_{k j}-v \leq 0
\end{aligned}
$$




$$
\begin{aligned}
& j=1, \cdots, n, \quad j \neq o, \\
& -\sum_{r=1}^{s} u_{r} y_{r o}^{U p}+z_{o}^{y} \gamma_{o}^{y}+\sum_{r=1}^{s} p_{r o}+\sum_{k=1}^{l} d_{k} b_{k o}^{L p}+z_{o}^{b} \gamma_{o}^{b} \\
& +\sum_{k=1}^{l} q_{k o}+v \geq 0 \\
& z_{j}^{y}+p_{r j} \geq u_{r}\left(y_{r j}^{U p}-y_{r j}^{L p}\right), \\
& r=1, \cdots, s, \quad j=1, \cdots, n, \\
& z_{j}^{b}+q_{k j} \geq d_{k}\left(b_{k j}^{U p}-b_{k j}^{L p}\right), \\
& k=1, \cdots, l, \quad j=1, \cdots, n, \\
& \theta_{o}^{R} \leq 1 \\
& z_{j}^{y} \geq 0, \quad z_{j}^{b} \geq 0, \quad j=1, \cdots, n, \\
& p_{r j} \geq 0, \quad q_{k j} \geq 0, \quad r=1, \cdots, s, \\
& k=1, \cdots, l, \quad j=1, \cdots, n, \\
& u_{r} \geq 0, \quad d_{k} \geq 0, \quad r=1, \cdots, s, \\
& k=1, \cdots, l .
\end{aligned}
$$

DMU at time $t$ and the frontier at time $t+1$ :

$$
\begin{gathered}
\left.y_{o}^{U q}, b_{o}^{L q} \mid q, p=t, t+1, p \neq q\right)=\min -\sum_{r=1}^{s} u_{r} y_{r_{o}}^{U q} \\
+z_{o}^{y} \gamma_{o}^{y}+\sum_{r=1}^{s} p_{r o}+\sum_{k=1}^{l} d_{k} b_{k o}^{L q}+z_{o}^{b} \gamma_{o}^{b} \\
+\sum_{k=1}^{l} q_{k o}+v
\end{gathered}
$$

s.t.:

$$
\begin{aligned}
& \sum_{r=1}^{s} u_{r} g_{y}+\sum_{k=1}^{l} d_{k} g_{b}=1 \\
& \sum_{r=1}^{s} u_{r} y_{r j}^{L p}+z_{j}^{y} \gamma_{j}^{y}+\sum_{r=1}^{s} p_{r j}-\sum_{k=1}^{l} d_{k} b_{k j}^{U p}+z_{j}^{b} \gamma_{j}^{b} \\
& \quad+\sum_{k=1}^{l} q_{k j}-v \leq 0 \\
& \quad j=1, \cdots, n, \quad j \neq o,
\end{aligned}
$$

$$
\begin{aligned}
& -\sum_{r=1}^{s} u_{r} y_{r o}^{U q}+z_{o}^{y} \gamma_{o}^{y}+\sum_{r=1}^{s} p_{r o}+\sum_{k=1}^{l} d_{k} b_{k o}^{L q}+z_{o}^{b} \gamma_{o}^{b} \\
& +\sum_{k=1}^{l} q_{k o}+v \geq 0, \\
& z_{j}^{y}+p_{r j} \geq u_{r}\left(y_{r j}^{U p}-y_{r j}^{L p}\right), \\
& r=1, \cdots, s, \quad j=1, \cdots, n, \\
& z_{j}^{b}+q_{k j} \geq d_{k}\left(b_{k j}^{U p}-b_{k j}^{L p}\right), \\
& k=1, \cdots, l, \quad j=1, \cdots, n, \\
& \theta_{o}^{R} \leq 1 \\
& z_{j}^{y} \geq 0, \quad z_{j}^{b} \geq 0, \quad j=1, \cdots, n, \\
& p_{r j} \geq 0, \quad q_{k j} \geq 0, \quad r=1, \cdots, s, \\
& k=1, \cdots, l, \quad j=1, \cdots, n, \\
& u_{r} \geq 0, \quad d_{k} \geq 0, \quad r=1, \cdots, s, \\
& k=1, \cdots, l \text {. }
\end{aligned}
$$

Note that all theorems with regard to Model (26) are proven; similarly, all of them can also be proven for Model (27).

Theorem 2. Proving Model (26) is always feasible.

Proof. We consider $z_{j}=0(j=1, \cdots, n), \gamma_{j}^{y}=$ $\gamma_{j}^{b}=0(j=1, \cdots, n)$, and $p_{r j}=q_{k j}=0(\forall r, k, j=$ $1, \cdots, n)$. Since $\nu \in R$, we can suppose that $\nu=1$. In addition, it is considered here that $d_{k}=0 \forall k$, $u_{r}=\frac{1}{y_{r_{o}}} \forall j=o$, and $u_{r}=0 \forall j \neq o$; it must be mentioned that $g_{y}=y_{r o}^{U p}$ is in Constraint (26a). By substituting these assumptions in Constraint (26a), we have:

$$
\sum_{r=1}^{s} \frac{1}{y_{r o}^{U p}} y_{r o}+0=1
$$

which always holds.

By substituting the above equation into constraint (26b), we have:

$$
0-v \leq 0, \quad j=1, \cdots, n \quad j \neq o,
$$

which always holds for $\nu=1$. The obtained solution satisfies Constraint (26c). Since $\gamma_{j}^{y}=\gamma_{j}^{b}=0(j=$ $1, \cdots, n)$, we can add to Constraints (26d) and (26e) the following: 


$$
\begin{aligned}
& y_{r j}^{U p}-y_{r j}^{L p}=0, \quad \forall r, j=1, \cdots, n, \\
& b_{k j}^{U p}-b_{k j}^{L p}=0, \quad \forall k, j=1, \cdots, n .
\end{aligned}
$$

Consequently, Constraints (26c) and (26d) hold. Since the value of the objective function in the obtained solution equals 1, Constraint (26f) holds, thus completing the proof.

Definition 9. In Models (26) and (27), $D M U_{o}$ is efficient if $\phi_{o}^{R p}=1$.

Conclusion 4. The efficiency scores of Models (26) and (27) are given by $\phi_{o}^{R p}=\frac{1}{1+\theta_{o}^{R p}}$.

Conclusion 5. Calculation of the MPI using the robust approach is as follows:

$$
\begin{aligned}
M^{R} & =\sqrt{\frac{\phi_{v}^{R t}\left(y_{o}^{t+1}, b_{o}^{t+1}\right)}{\phi_{v}^{R t}\left(y_{o}^{t}, b_{o}^{t}\right)} \times \frac{\phi_{v}^{R t+1}\left(y_{o}^{t+1}, b_{o}^{t+1}\right)}{\phi_{v}^{R t+1}\left(y_{o}^{t}, b_{o}^{t}\right)}} \\
& \times \sqrt{\frac{S E^{R t}\left(y_{o}^{t+1}, b_{o}^{t+1}\right)}{S E^{R t}\left(y_{o}^{t}, b_{o}^{t}\right)} \times \frac{S E^{R t+1}\left(y_{o}^{t+1}, b_{o}^{t+1}\right)}{S E^{R t+1}\left(y_{o}^{t}, b_{o}^{t}\right)}}
\end{aligned}
$$

Theorem 3. Prove $M^{R} \leq M^{U}$.

Proof. The values of objective function for Models (26) and (13) are given by the following two relations:

$$
\begin{aligned}
& -\sum_{r=1}^{s} u_{r} y_{r o}^{U}+z_{o}^{y} \gamma_{o}^{y}+\sum_{r=1}^{s} p_{r o}+\sum_{k=1}^{l} d_{k} b_{k o}^{L}+z_{o}^{b} \gamma_{o}^{b} \\
& \quad+\sum_{k=1}^{l} q_{k o}+v
\end{aligned}
$$

and:

$$
-\sum_{r=1}^{s} y_{r o}^{U} u_{r}+\sum_{k=1}^{l} b_{k o}^{L} d_{k}+v .
$$

Since $p_{r}, q_{k}, z_{o}^{y}, z_{o}^{b}, \gamma_{o}^{y}, \gamma_{o}^{b}$ are positive, Eq. (32) is greater than Eq. (33). Thus, efficiency scores $\phi_{o}^{R p}$ in Models (26) and (27) are smaller than efficiency scores $\phi_{o}^{U p}$ in Models (13) and (14). Hence, it becomes obvious that $M^{R} \leq M^{U}$.

Algorithm 1 summarizes computing the MPI of the DMUs with undesirable outputs and data uncertainty according to the directional distance function.

\section{Numerical example}

In order to demonstrate the applicability of the considered methods, a simple example including 5 DMUs, each of which produces one desirable and one undesirable interval output, is expressed in Subsection 4.1. In addition, a case study of data related to the National Bank branches in Ardabil, Iran is investigated in Subsection 4.2. Then, in Subsection 4.3, the results of Aghayi and Maleki [40] and those obtained from the proposed models will be compared.

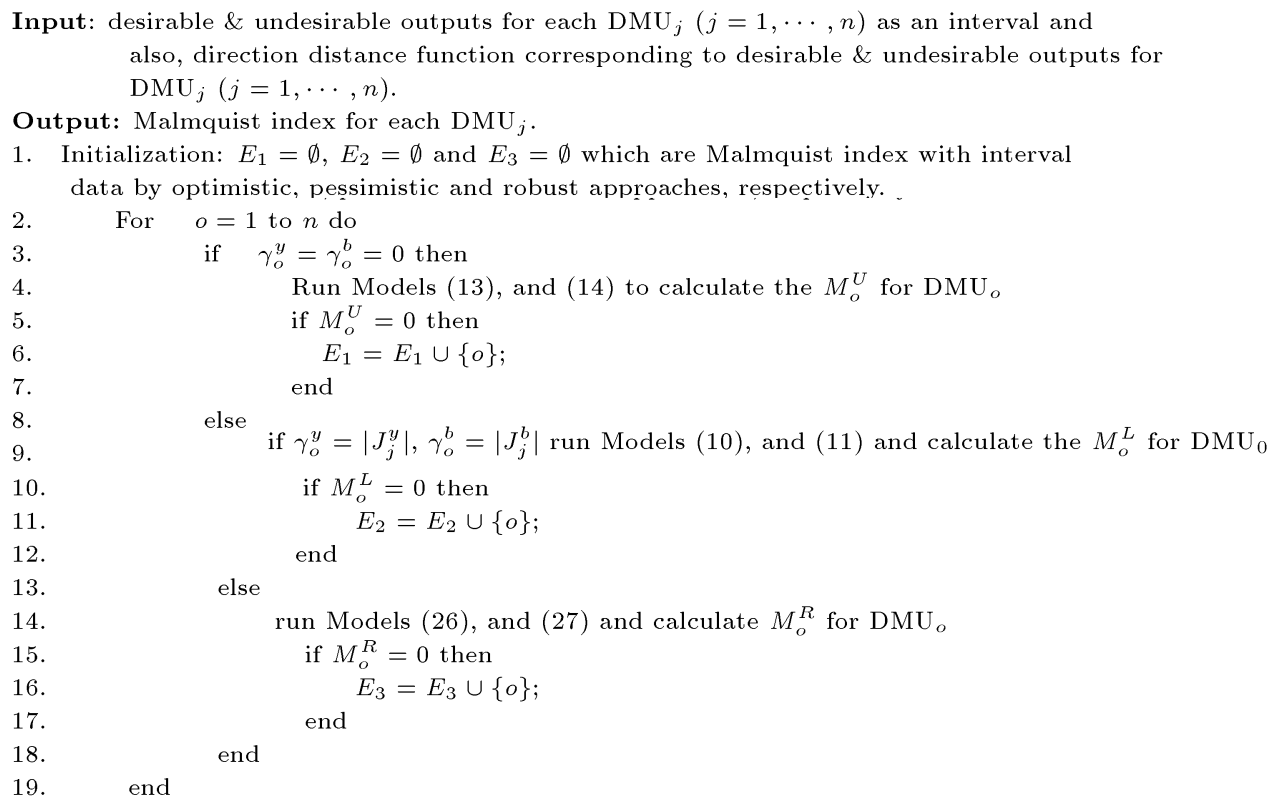

Algorithm 1. The MPI of the DMUs based on the directional distance function. 


\subsection{Simple example}

Here, 5 DMUs are considered, each of which with two interval outputs-one desirable, one undesirable-in time periods $t$ and $t+1$, as shown in Tables 1 and 2 .

The results of solving Models (3), (4), (10), (11), (13), (14), (26), and (27) are represented in Table 3.

According to Table 3, all DMUs fall into the sixth category; for all cases, the productivity rise in the optimistic scenario is greater than that in the pessimistic scenario. Having efficiency scores smaller than one under both the optimistic and pessimistic scenarios, only $D M U_{2}$ belongs to the third category. In the optimistic scenario, the DMUs can be ranked in terms of productivity progress as $D M U_{3}>D M U_{1}>D M U_{4}$, $D M U_{5}>D M U_{2}$. Under the pessimistic scenario, the DMUs are ranked based on productivity regress as $D M U_{1}>D M U_{3}>D M U_{2}>D M U_{4}>D M U_{5}$. It is worth mentioning that the order of productivity rise may be different from that of productivity decline. Since one desirable output and one undesirable output are dealt with in this simple numerical example, $\gamma_{o}^{y}=1$ and $\gamma_{o}^{b}=1$ when the robust approach is implemented. In order to calculate $\theta_{j}^{R p}$, the average value of efficiency is divided into two decimal places for each $\gamma$. In

Table 1. Data related to the desirable outputs in time periods $t$ and $t+1$.

\begin{tabular}{ccccc}
\hline DMU & $\boldsymbol{y}_{j}^{\boldsymbol{l t}}$ & $\boldsymbol{y}_{j}^{\boldsymbol{l t + 1}}$ & $\boldsymbol{y}_{j}^{\boldsymbol{u} \boldsymbol{t}}$ & $\boldsymbol{y}_{j}^{\boldsymbol{u}+\mathbf{1}}$ \\
\hline 1 & 5 & 10 & 9 & 13 \\
2 & 5 & 10 & 9 & 11 \\
3 & 15 & 20 & 21 & 22 \\
4 & 10 & 15 & 20 & 20 \\
5 & 30 & 35 & 31 & 37 \\
\hline
\end{tabular}

Table 2. Data pertaining to undesirable outputs in time periods $t$ and $t+1$.

\begin{tabular}{ccccc}
\hline DMU & $\boldsymbol{b}_{j}^{\boldsymbol{l t}}$ & $\boldsymbol{b}_{j}^{\boldsymbol{l t + 1}}$ & $\boldsymbol{b}_{\boldsymbol{j}}^{\boldsymbol{u} \boldsymbol{t}}$ & $\boldsymbol{b}_{j}^{\boldsymbol{u t + 1}}$ \\
\hline 1 & 5 & 10 & 8 & 15 \\
2 & 15 & 20 & 20 & 25 \\
3 & 20 & 25 & 22 & 30 \\
4 & 40 & 45 & 42 & 50 \\
5 & 25 & 30 & 27 & 35 \\
\hline
\end{tabular}

Table 3. Results of solving Models (3), (4), (10), (11), (13), (14), (26), and (27).

\begin{tabular}{ccccc}
\hline DMU & $\boldsymbol{M}_{\boldsymbol{j}}$ & $\underline{\underline{M}}_{j}$ & $\overline{\boldsymbol{M}}_{\boldsymbol{j}}$ & $\boldsymbol{M}_{\boldsymbol{j}}^{\boldsymbol{R}}$ \\
\hline 1 & 0.98 & 0.66 & 1.32 & 1 \\
2 & 1.07 & 0.81 & 0.95 & 0.94 \\
3 & 1.00 & 0.75 & 1.47 & 0.92 \\
4 & 1.09 & 0.85 & 1.25 & 0.94 \\
5 & 1 & 0.90 & 1.25 & 1 \\
\hline
\end{tabular}

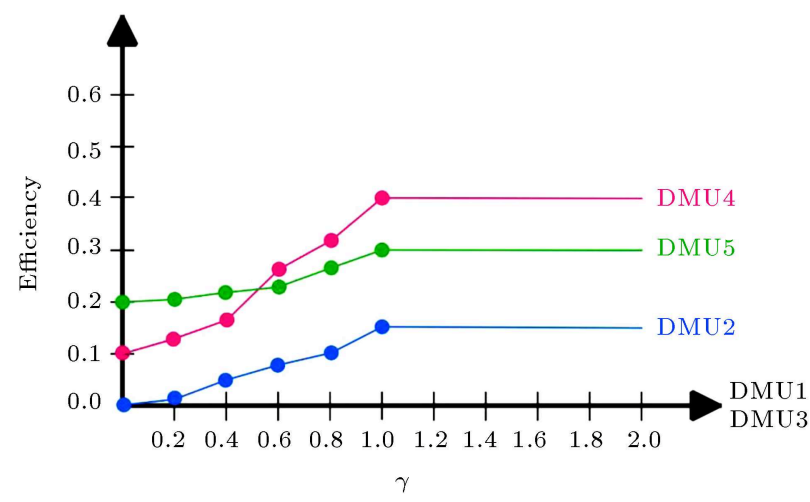

Figure 1. Efficiency values of the DMUs for various $\gamma$ values.

addition, this example considered $\gamma \in[0,2]$ with a step length of 0.2 . In this regard, it is assumed that there are two random parameters: for simplification, $\gamma=\gamma_{o}^{y}+\gamma_{o}^{b}$. Based on the comparison of the results for $M_{j}^{R}$ in Table 3 , it is observed that there is no change in productivity at time $t+1$ as compared to productivity at time $t$, because $M_{1}^{R}, M_{5}^{R}=1$. Furthermore, $M_{2}^{R}, M_{3}^{R}, M_{4}^{R}<1$ implies productivity regress.

Considering the two random parameters simplified in the following form: $\gamma=\gamma_{o}^{y}+\gamma_{o}^{b}$, Figure 1 shows efficiency changes for different values of $\gamma$. As observed in Figure 1, the maximum coincidence occurs for $\gamma \in[1,2]$, that is to say, data fluctuations do not affect efficiency when the value of $\gamma$ is equal to or greater than 1 .

\subsection{Case study}

Nowadays, the significant role of financial institutions is no secret to anyone. In most countries, banks play an integral role in this regard; they affect the economic performance of countries through mobilizing resources, providing means of payment, granting facilities, and creating interactions between investment and saving. Since the most important mission of the banking system is the collection and optimal allocation of public funds to productive economic activities, the volume of granted facilities in return for a specific level of used inputs and deposits remains one of the primary criteria for evaluating the proper performance of each bank. The application of the MPI proves to be one of the main methods of evaluating the performance of banks. In fact, higher efficiency and productivity of the banking industry in any country is synonymous with lower banking costs, higher interest rates, and superior service quality, leading ultimately to a decrease in investment costs. In order to show that the proposed methods herein are applicable to real-world environments, where interval data and undesirable outputs are dealt with, the efficiency of 30 branches of the National Bank in Ardabil Province, Iran over a 4-year period from 2011 to 2014 was evaluated. 
With this end in view, each branch of National Bank was considered a DMU with constant inputs, such as the terms and values of deposits required for getting loans and the rate of interest for each loan, which are all fixed and identical in all branches of the National Bank of Iran. This study adopts an intermediate approach, whereby financial institutions such as banks are merely financial intermediaries and assume two major roles of receiving and distributing resources in the economy. By virtue of this approach, we will define an indicator for desirable outputs, which are revenue per branch. Moreover, non-performing loans and the amount of four main types of deposits-i.e., interest-free deposits, short-term investment deposits, long-term investment deposits, and current deposits-are defined as indicators of undesirable outputs. Since we always treat inputs as undesirable outputs, the amount of deposits is taken as an undesirable index. In order to evaluate the efficiency of these branches, Models (10), (11), (13), and (14) are first employed for efficiency measurement under optimistic and pessimistic scenarios and, then, the branches are divided into the six categories mentioned above. Then, for finding a deterministic solution, the proposed robust model is used. According to Table 4, the last column includes values of efficiency obtained by executing Model (13). For calculating this column, the average values achieved through solving Models (26) and (27) for a step length of 0.2 have been used. In addition, because there are one desirable and two undesirable outputs in this evaluation, $\gamma \in[0,3]$. In comparison with other similar models, the advantage of using the robust model is that its results are of high validity since it is calculated in the worst-case scenario. $D M U_{2}, D M U_{3}$, and $D M U_{21}$ exhibit progress because $M_{2}^{R}, M_{3}^{R}, M_{21}^{R}>1 ; M_{4}^{R}$, $M_{5}^{R}, M_{22}^{R}, M_{23}^{R}, M_{24}^{R}=1$; therefore, nothing can be said about these DMUs; the other DMUs have regress. It can be seen that $M_{j}^{L} \leq M_{j}^{U}$ and $M_{j}^{R} \leq$ $M_{j}^{U}$, reflecting the fact that the obtained results are completely consistent with the proven theorems herein. The results of calculating the MPI of the considered National Bank branches of Iran are demonstrated in Figure 2.

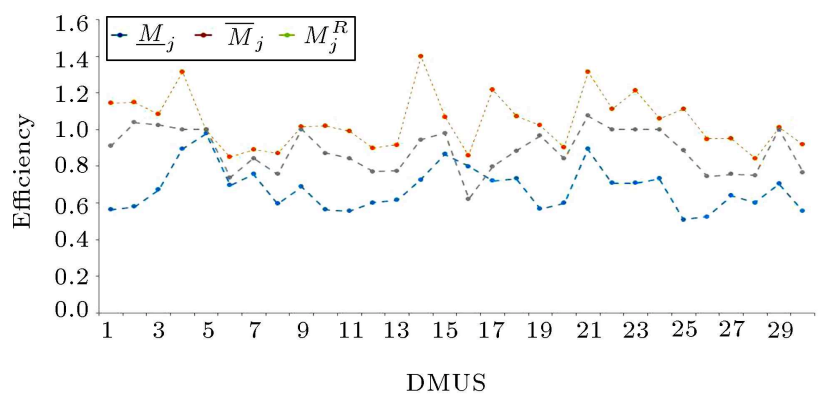

Figure 2. Comparison of the results by calculating the MPI of the National Bank of Iran branches.
Table 4. Results of the MPI of National Bank of Iran branches.

\begin{tabular}{|c|c|c|c|c|}
\hline DMU & $\underline{M}_{j}$ & $\bar{M}_{j}$ & Justification & $M_{j}^{R}$ \\
\hline 1 & 0.563065 & 1.14586 & $E$ & 0.910306 \\
\hline 2 & 0.578326 & 1.149271 & $E$ & 1.03923 \\
\hline 3 & 0.671972 & 1.082658 & $E$ & 1.024695 \\
\hline 4 & 0.892928 & 1.314586 & $E$ & 1 \\
\hline 5 & 0.978419 & 1 & $E-$ & 1 \\
\hline 6 & 0.694989 & 0.849843 & $E--$ & 0.737387 \\
\hline 7 & 0.756402 & 0.889302 & $E--$ & 0.843447 \\
\hline 8 & 0.59453 & 0.8721 & $E--$ & 0.755794 \\
\hline 9 & 0.687906 & 1.016441 & $E$ & 1 \\
\hline 10 & 0.562258 & 1.020162 & $E$ & 0.872228 \\
\hline 11 & 0.554886 & 0.989638 & $E--$ & 0.843412 \\
\hline 12 & 0.601147 & 0.897519 & $E--$ & 0.769528 \\
\hline 13 & 0.615545 & 0.916612 & $E--$ & 0.774725 \\
\hline 14 & 0.726207 & 1.40005 & $E$ & 0.943396 \\
\hline 15 & 0.864611 & 1.068994 & $E$ & 0.980392 \\
\hline 16 & 0.798679 & 0.857059 & $E--$ & 0.620269 \\
\hline 17 & 0.720033 & 1.216943 & $E$ & 0.798111 \\
\hline 18 & 0.732865 & 1.070131 & $E$ & 0.882346 \\
\hline 19 & 0.566529 & 1.023449 & $E$ & 0.966736 \\
\hline 20 & 0.59957 & 0.903914 & $E--$ & 0.84112 \\
\hline 21 & 0.893314 & 1.314533 & $E$ & 1.075905 \\
\hline 22 & 0.707107 & 1.112718 & $E$ & 1 \\
\hline 23 & 0.707107 & 1.211172 & $E$ & 1 \\
\hline 24 & 0.731272 & 1.058372 & $E$ & 1 \\
\hline 25 & 0.508698 & 1.112751 & $E$ & 0.884956 \\
\hline 26 & 0.525051 & 0.948891 & $E--$ & 0.74511 \\
\hline 27 & 0.638628 & 0.95121 & $E--$ & 0.755882 \\
\hline 28 & 0.601131 & 0.840623 & $E--$ & 0.750766 \\
\hline 29 & 0.704135 & 1.012594 & $E$ & 1 \\
\hline 30 & 0.554469 & 0.917087 & $E--$ & 0.76716 \\
\hline
\end{tabular}

\subsection{Comparison with the paper authored by Aghayi and Maleki [40]}

In this section, the results obtained by applying the proposed robust Malmquist model-which is based on the Directional Distance Function (DDF) and is also effective in the presence of undesirable outputs-to 30 branches of the National Bank of Iran across Ardabil, Iran, are compared with those obtained from the DDF-based robust model proposed by Aghayi and Maleki [40]. The results of executing the DDF-based robust model for a step length of 0.2 are given in Table 5. Considering the fact that, in this evaluation, we deal with one desirable and two undesirable outputs, $\gamma \in[0,3]$. These results have been produced by analyzing data from 2011 to 2014. Datasets related to 2011 and 2014 have been taken as the lower and 
Table 5. Results of the comparison between the proposed method and Aghayi and Maleki's method [40].

\begin{tabular}{|c|c|c|}
\hline DMU & $\phi_{j}^{R}$ & $M_{j}^{R}$ \\
\hline 1 & 1 & 0.910306 \\
\hline 2 & 1 & 1.03923 \\
\hline 3 & 1 & 1.024695 \\
\hline 4 & 1 & 1 \\
\hline 5 & 1 & 1 \\
\hline 6 & 1 & 0.737387 \\
\hline 7 & 0.84 & 0.843447 \\
\hline 8 & 1 & 0.755794 \\
\hline 9 & 1 & 1 \\
\hline 10 & 1 & 0.872228 \\
\hline 11 & 1 & 0.843412 \\
\hline 12 & 1 & 0.769528 \\
\hline 13 & 1 & 0.774725 \\
\hline 14 & 1 & 0.943396 \\
\hline 15 & 1 & 0.980392 \\
\hline 16 & 0.81 & 0.620269 \\
\hline 17 & 0.93 & 0.798111 \\
\hline 18 & 0.59 & 0.882346 \\
\hline 19 & 1 & 0.966736 \\
\hline 20 & 1 & 0.84112 \\
\hline 21 & 0.92 & 1.075905 \\
\hline 22 & 1 & 1 \\
\hline 23 & 1 & 1 \\
\hline 24 & 0.99 & 1 \\
\hline 25 & 1 & 0.884956 \\
\hline 26 & 1 & 0.74511 \\
\hline 27 & 1 & 0.755882 \\
\hline 28 & 1 & 0.750766 \\
\hline 29 & 1 & 1 \\
\hline 30 & 1 & 0.76716 \\
\hline
\end{tabular}

upper bounds, respectively. As shown in Table 5, the number of efficient units according to the DDF-based robust model proposed by Aghayi and Maleki [40] is too high, stressing the need for ranking efficient DMUs. Yet, the results of the DDF-based robust Malmquist model demonstrate that over a four-year period from 2011 to 2014, only 3 units exhibited progress, 7 units did not have progress, and other units showed regress. Moreover, Figure 3 compares the results of the DDF-

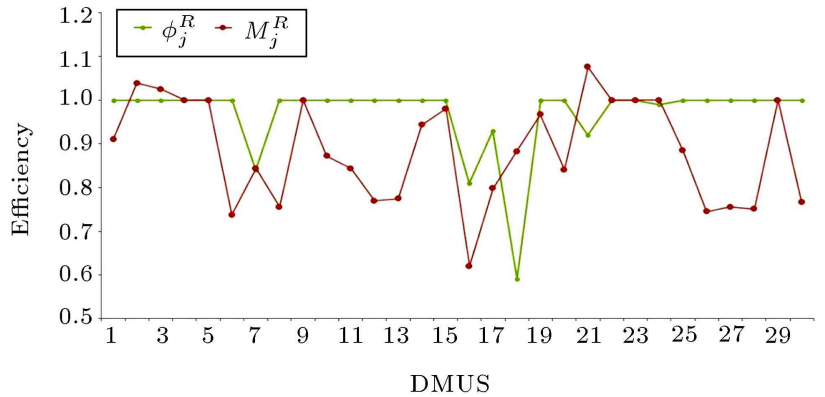

Figure 3. Comparison of the results in this paper with those in Aghayi and Maleki's method [2].

based robust Malmquist model with those obtained by the DDF-based robust model proposed by Aghayi and Maleki [40].

\section{Conclusion}

Data uncertainty is one of the main concerns of industrial, economic, and manufacturing planners. Thus, it is important to achieve robust optimal solutions to these problems. In the current paper, the MPI and the DEA technique were used to investigate the efficiency status and productivity changes of the branches of the National Bank of Iran across Ardabil, Iran, during four consecutive years. Initially, the present paper considered the interval-data of Malmquist model based on the directional distance function with undesirable outputs; by increasing the desirable outputs and decreasing the undesirable outputs, it simultaneously evaluated the efficiency values. Then, it was shown that the efficiency value was not certain and, instead, depended on an interval. Next, in order to assess the DMUs, they were divided into six categories. After that, the DDF-based robust Malmquist model was presented in the presence of undesirable outputs, without increasing the problem complexity. The proposed model aimed to minimize the maximum value of the objective function with the optimization of the worst-case scenario. Moreover, by defining a conservatism level, the feasibility of optimal solution was guaranteed. The biggest advantage of the considered robust model is that since the conservatism degree is adjustable, the proposed method performs conservatively and provides a deterministic solution. Designing a robust model for calculating the MPI of the DUMs with undesirable outputs along with fuzzy and negative data is suggested for future researches.

\section{Acknowledgement}

The research was carried out during a sabbatical stay by Nazila Aghayi at the Universite Catholique de Louvain. Financial support by Ardabil branch, Islamic Azad University, Ardabil, Iran is gratefully acknowledged. 


\section{References}

1. Farrell, M.J. "The measurement of productive efficiency", Journal of the Royal Statistical Society, 120(3), pp. 253-281 (1957)

2. Charnes, A., Cooper, W.W., and Rhodes, E. "Measuring the efficiency of decision making units", European Journal of Operational Research, 2(6), pp. 429-444 (1978).

3. Banker, R.D., Charnes, A., and Cooper, W.W. "Some models for estimating technical and scale inefficiencies in data envelopment analysis", Management Science, 30(9), pp. 1078-1092 (1984).

4. Pittman, R.W. "Multilateral productivity comparisons with undesirable outputs", Economic Journal, 93(372), pp. 883-891 (1983).

5. Caves, D.W., Christensen, L.R., and Diewert, E. "Multilateral comparisons of output, input and productivity using superlative index numbers", The Economic Journal, 92(365), pp. 73-86 (1982).

6. Ardabili, J.S., Aghayi, N., and Monzali, A.L. "New efficiency using undesirable factors of data envelopment analysis", Modeling \& Optimization, 9(2), pp. 249-255 (2007).

7. Malmquist, S. "Index numbers and indifference surfaces", Trabajos de Estatistica, 4(2), pp. 209- 242 (1953).

8. Fare, R., Grosskopf, S., and Logan, J. "The relative efficiency of Illinois electric utilities", Resources and Energy, 5, pp. 349-367 (1983).

9. Soyster, A.L. "Convex programming with set- inclusive constraints and applications to inexact linear programming", Operational Research, 21, pp. 1154-1157 (1972).

10. Seiford, L.M. and Zhu, J. "Modeling undesirable factors inefficiency valuation", European Journal of Operational Research, 142(1), pp. 16-20 (2002).

11. Chambers, R.G., Chung, Y., and Fare, R. "Benefit and distance function", Journal of Economic Theory, 70(2), pp. 407-419 (1996).

12. Chung, Y.H., Fare, R., and Grosskopf, S. "Productivity and undesirable outputs a directional distance function approach", Journal of Environmental Management, 51(3), pp. 229-240 (1997).

13. Shepherd, R.W., Theory of Cost and Production Functions, Princeton, NJ, USA: Princeton University press (1970).

14. Zanella, A., Camanho, A., and Dias, T. "Undesirable outputs and weighting schemes in composite indicators based on data envelopment analysis", European Journal of Operational Research, 245, pp. 517-530 (2015).

15. Iftikhar, Y., Wang, Z., Zhang, B., and Wang, B. "Energy and $\mathrm{CO}_{2}$ emissions efficiency of major economies: A network DEA approach", Energy, 147, pp. 197-207 (2018).
16. Khoshandam, L., Kazemi, R., and Amirteimoori, A. "Marginal rate of substitution in data envelopment analysis with undesirable outputs: A directional approach", Measurement, 68, pp. 49-57 (2015).

17. Barnabé, W. "Disaggregation of the cost Malmquist productivity index with joint and output-specific inputs", Omega, 75, pp. 1-12 (2018).

18. Sueyoshi, T., Goto, M., and Wang, D. "Malmquist index measurement for sustainability enhancement in Chinese municipalities and provinces", Energy Economics, 67, pp. 554-571 (2017).

19. Sueyoshi, T. and Goto, M. "DEA environmental assessment in time horizon: Radial approach Malmquist index measurement on petroleum companies", Energy Economics, 51, pp. 329-345 (2015).

20. Fuentes, R. and Lillo-Banuls, A. "Smoothed bootstrap Malmquist index based on DEA model to compute productivity of tax offices", Expert Systems with Applications, 42, pp. 2442-2450 (2015).

21. Yu, C., Shi, L., Wang, Y., Chang, Y., and Cheng, B. "The eco-efficiency of pulp and paper industry in China: an assessment based on slacks-based measure and Malquist-Luenberger index", Journal of Cleaner Production, 127, pp. 511-521 (2016).

22. Kao, C. "Measurement and decomposition of the Malmquist productivity index for parallel production systems", Omega, 67, pp. 54-59 (2016).

23. Maroto, A. and Zofio, J. "Accessibility gains and road transport infrastructure in Spain: A productivity approach based on the Malmquist index", Journal of Transport Geography, 52, pp. 143-152 (2016).

24. Emrouznejad, A., Rostamy-Malkhalifeh, M., HatamiMarbini, A., Tavana, M., and Aghayi, N. "An overall profit Malmquist productivity index with fuzzy and interval data", Mathematical and Computer Modelling, 54(11-12), pp. 2827-2838 (2011).

25. Wanke, P., Barros, C.P., and Emrouznejad, A. "Assessing productive efficiency of banks using integrated Fuzzy-DEA and bootstrapping a case of Mozambican banks", European Journal of Operational Research, 249(1), pp. 378-389 (2016).

26. Mashayekhi, Z. and Omrani, H. "An integrated multi objective Markowitz-DEA cross efficiency model with fuzzy returns for portfolio selection problem", Operation Research, 38, pp. 1-9 (2016).

27. Aghayi, N. "Cost efficiency measurement with fuzzy data in DEA", Journal of Intelligent and Fuzzy Systems, 32, pp. 409-420 (2017).

28. Toloo, M., Aghayi, N., and Rostamy-Malkhalifeh, M. "Measuring overall profit efficiency with interval data", Applied Mathematics and Computation, 201(1-2), pp. 640-649 (2008). 
29. Hatami-Marbini, A., Emrouznejad, A., and Agrell, P. "Interval data without sign restrictions in DEA", Applied Mathematical Modelling, 38(7-8), pp. 20282036 (2014).

30. Salehpour, S. and Aghayi, N. "The most revenue efficiency with price uncertainty", International Journal of Data Envelopment Analysis, 3, pp. 575-592 (2015).

31. Kouvelis, P. and Yu, G., Robust Discrete Optimization and Its Applications, Kluwer Academic publishers Norwell, MA (1997).

32. Ben-Tall, A. and Nemirovski, A. "Robust convex optimization", Mathematical Operation Research, 23, pp. 769-805 (1998).

33. El-Ghaoui, L. and Lebret, H. "Robust solutions to least-squares problems to uncertain data matrices", Sima Journal on Matrix Analysis and Applications, 18, pp. 1035-1064 (1997).

34. Bertsimas, D. and Sim, M. "The price of the robustness", Operation Research, 52, pp. 35-53 (2004).

35. Zahedi-Seresht, M., Jahanshahloo, G.R., and Jablonsky, J. "A robust data envelopment analysis model with different scenarios", Applied Mathematical Modelling, 52, pp. 306-319 (2017).

36. Yousefi, S., Soltani, R., Saen, R.F., and Pishvaee, M.S. "A robust fuzzy possibilistic programming for a new network GP-DEA model to evaluate sustainable supply chains", Journal of Cleaner Production, 166, pp. 537-549 (2017).

37. Chung-Cheng, L. "Robust data envelopment analyses approaches for evaluating algorithmic performance", Computers and Industrial Engineering, 81, pp. 78-89 (2015).

38. Mardani, M. and Salarpour, M. "Measuring technical efficiency of potato production in Iran using robust data envelopment analysis", Information Processing in Agriculture, 2(1), pp. 6-14 (2015).

39. Aghayi, N., Tavana, M., and Raayatpanah, M.A. "Robust efficiency measurement with common set of weights under varying degrees of conservatism and data uncertainty", European Journal of Industrial Engineering, 10(30), pp. 385-405 (2016).

40. Aghayi, N. and Maleki, B. "Efficiency measurement of DMUs with undesirable outputs under uncertainty based on the directional distance function: Application on Bank Industry", Energy, 112, pp. 376-387 (2016).

41. Ray, C. and Desli, E. "Productivity growth, technical progress, and efficiency change in industrialized countries: comment", The American Economic Review, 87, pp. 1033-1039 (1997).

\section{Biographies}

Nazila Aghayi is an Assistant Professor at the Department of Mathematics in Islamic Azad University of Ardabil, Ardabil, Iran. She received her PhD in Operational Research from Science and Research Branch, Islamic Azad University, Tehran, Iran in 2013. She was in Center of Operations Research and Econometrics of UCL University for a sabbatical position in 2017. She is the author of several scientific publications in the area of Operations Research and Data Envelopment Analysis. She has published many papers in international scholarly academic journals. Her areas of interest are operations research, data envelopment analysis, computer science, fuzzy system, and robust optimization.

Madjid Tavana is a Professor and the Distinguished Chair of Business Analytics at La Salle University, where he serves as Chairman of the Business Systems and Analytics Department. He also holds an Honorary Professorship in Business Information Systems at the University of Paderborn in Germany. Dr. Tavana is a Distinguished Research Fellow at the Kennedy Space Center, the Johnson Space Center, the Naval Research Laboratory at Stennis Space Center, and the Air Force Research Laboratory. He was recently honored with the prestigious Space Act Award by NASA. He holds MBA, PMIS, and PhD degrees in Management Information Systems and received his Post-Doctoral Diploma in Strategic Information Systems from the Wharton School at the University of Pennsylvania. He has published 13 books and over 250 research papers in international scholarly academic journals. $\mathrm{He}$ is the Editor-in-Chief of International Journal of Applied Decision Sciences, International Journal of Management and Decision Making, International Journal of Communication Networks and Distributed Systems, International Journal of Knowledge Engineering and Data Mining, International Journal of Strategic Decision Sciences, and International Journal of Enterprise Information Systems.

Bentolhoda Maleki received her MSc in Mathematics from Islamic Azad University of Ardabil, Ardabil, Iran in 2016. She participated in many conferences and scientific events. She published her papers in Energy and International Journal of Data Envelopment Analysis. 